\title{
Application and Drying of Automotive Paints
}

\author{
Dr. Sanjeev Chandra \\ Centre for Advanced Coating Technologies \\ Department of Mechanical and Industrial Engineering \\ University of Toronto
}

Paint on the exterior of an automobile has to look attractive and also serve as a functional coating that resists corrosion and abrasion over a service life of many years. Paints are applied using electrostatic rotating bell atomizers in which a thin paint film is forced onto the inner surface of a high-speed rotating bell where it breaks into droplets that are directed onto the target surface by air jets and electrostatic forces. Painted component are dried in a heated oven where solvents evaporate leaving behind a resin that polymerizes to form a hard layer.

Experiments were done to study the formation of defects in paint films during both paint application and drying. Highspeed videos were taken of paint atomization in rotating bell atomizers and the impact of paint droplets on a solid surface. The effect of spray parameters on droplet size and the rate of surface coverage was measured. Painted samples were placed in an oven and while they dried the paint surface was photographed and their weight loss measured. Cellular structures appeared on the paint surface, induced by Marangoni flows driven by solvent concentration variations. For thin films $(<500$ $\mu \mathrm{m})$ the patterns disappeared in a few minutes and the dried paint surface became smooth while for thicker paint films rolllike structures remained on the hardened paint layer, creating an uneven surface commonly known as an "orange-peel" defect. Pigments and metallic particles that are commonly added to paints were observed to move due to surface-tension driven flows, forming clusters that could cause changes in colour. An analytical solution of the mass-diffusion equation was used to model solvent evaporation from the paint film and calculate the concentration gradient and surface tension variations in the paint films. Small air bubbles were trapped in the paint film under impacting droplets and grew larger as evaporating solvent diffuse into them, creating blisters. 